\title{
TEM Sample Preparation of Patterned Quantum Dots
}

\author{
H Wang ${ }^{1 *}$, V Srot $^{1}$, B Fenk ${ }^{1}$, G Laskin $^{1}, \mathrm{~J} \mathrm{Mannhart}^{1}$ and PA van Aken ${ }^{1}$ \\ ${ }^{1 .}$ Max Planck Institute for Solid State Research, Stuttgart, Germany. \\ * Corresponding author: hgwang@fkf.mpg.de
}

Quantum nanostructures offer many possibilities for artificial manipulation of novel electron systems which are absent in their bulk analogues [1]. Among them, quantum dots (QDs) have triggered much research attention due to their size confinement [2] and play an important role in novel applications like quantum computing and sensing [3]. For understanding the emergent properties of QDs, detailed scanning transmission electron microscope (STEM) investigations of their microstructure, interfaces and local chemistry at the nanoscale are important. The small size of the QDs makes site-specific TEM sample preparation exceptionally challenging.

In this work, we developed an optimized method for achieving high-quality TEM specimen consisting of $\mathrm{SrRuO}_{3}$ (SRO) QDs epitaxially grown on $\mathrm{SrTiO}_{3}$ (STO) substrate [4]. The top view of the $30 \mathrm{~nm}$ SRO QDs periodic array on STO substrate is shown in Figure 1. A focused ion beam (FIB) (Zeiss CrossBeam XB 1540) has been used to prepare the TEM lamella in cross-sectional orientation with several large electrontransparent regions that are separated by thicker walls (Fig. 1b). Using this approach, we effectively reduce the bending of the lamellae and enhance the number of SRO QDs, which are subsequently investigated by STEM techniques. Each electron-transparent area was thinned by FIB to different thicknesses, where the thinnest region was around $50 \mathrm{~nm}$ and the thickest around $70 \mathrm{~nm}$.

Final thinning and polishing of the targeted regions to remove amorphised material, implanted Ga ions, or redeposited material was performed using a NanoMill system (Fischione Model 1040). A cross-section through a complete single SRO QD structure on STO substrate acquired by aberration-corrected STEM equipped with a high-angle annular dark-field (HAADF) detector is shown in Figure 2. We demonstrate an efficient approach for the preparation of high-quality TEM specimens of QDs, which could have potential benefits for the preparation of different nanostructures and for their nanoscale investigations [5].

References:

[1] H Boschker et al., Annu. Rev. Conden. Ma. P. 8 (2017), p. 145.

[2] AP Alivisatos, Science 271 (1996), p. 933.

[3] P Senellart et al., Nat. Nanotech. 12 (2017), p. 1026.

[4] G Laskin et al., Nano Lett. (2019), in press.

[5] The research leading to these results has received financial support from the China Scholarship Council (No. 201504910813) and the Max Planck Society. This project has received funding from the European Union's Horizon 2020 research and innovation programme under grant agreement No. 823717 ESTEEM3. 

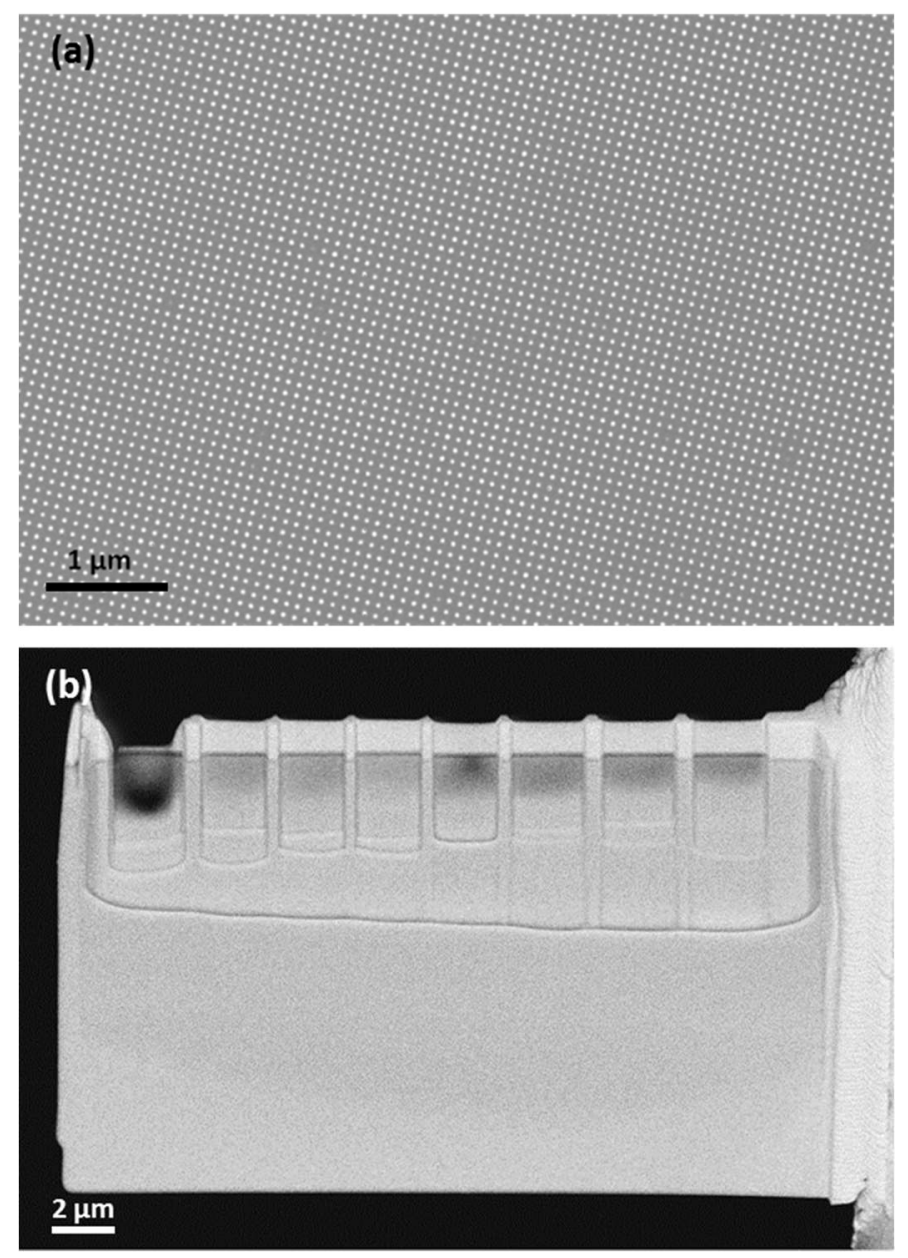

Figure 1. (a) An SEM image of the top-view of the patterned QD array. (b) An SEM image of a prepared FIB lamella taken by the energy-selective backscattered (EsB) detector.

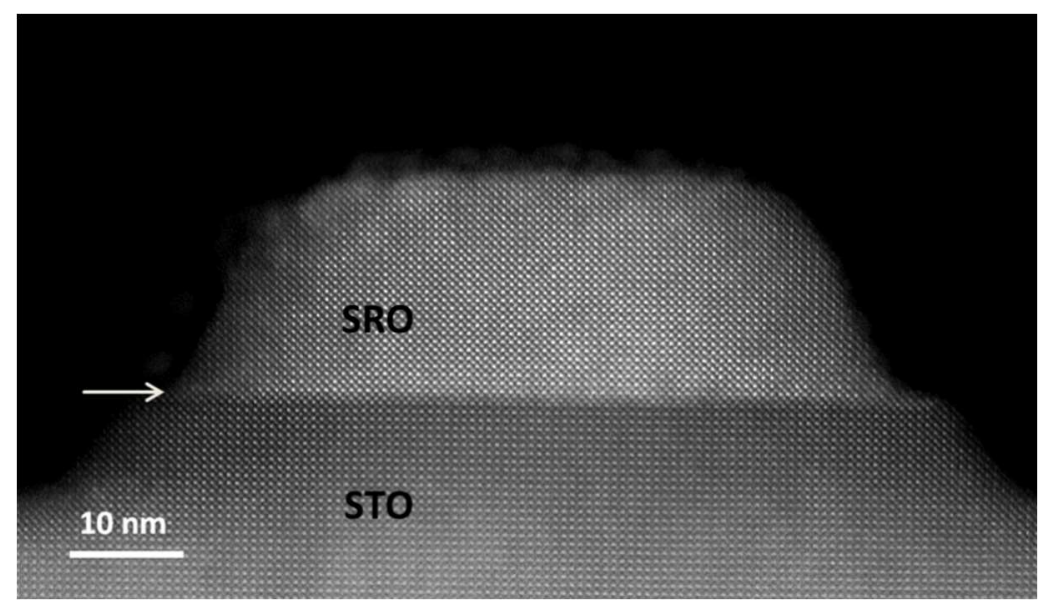

Figure 2. An HAADF-STEM image of a single SRO QD epitaxially grown on an STO substrate. The white arrow marks the interface between the SRO QD and the STO substrate. 